\title{
A!
}

This is an electronic reprint of the original article.

This reprint may differ from the original in pagination and typographic detail.

Tayyab, Muhammad; Gelabert, Xavier; Jäntti, Riku

\section{A Simulation Study on Handover in LTE Ultra-Small Cell Deployment: A 5G Challenge}

Published in:

2019 IEEE 2nd 5G World Forum (5GWF)

DOI:

10.1109/5GWF.2019.8911616

Published: 01/01/2019

Document Version

Peer reviewed version

Please cite the original version:

Tayyab, M., Gelabert, X., \& Jäntti, R. (2019). A Simulation Study on Handover in LTE Ultra-Small Cell Deployment: A 5G Challenge. In 2019 IEEE 2nd 5G World Forum (5GWF) (pp. 388-392). IEEE.

https://doi.org/10.1109/5GWF.2019.8911616

This material is protected by copyright and other intellectual property rights, and duplication or sale of all or part of any of the repository collections is not permitted, except that material may be duplicated by you for your research use or educational purposes in electronic or print form. You must obtain permission for any other use. Electronic or print copies may not be offered, whether for sale or otherwise to anyone who is not an authorised user. 


\section{A Simulation Study on Handover in LTE Ultra-Small Cell Deployment: A 5G Challenge}

\author{
M. Tayyab ${ }^{1,3}$ \\ ${ }^{1}$ Huawei Technologies Finland Oy \\ Helsinki, Finland \\ muhammad.tayyab5@huawei.com \\ muhammad.tayyab@aalto.fi
}

\author{
X. Gelabert ${ }^{2}$ \\ ${ }^{2}$ Huawei Technologies Sweden AB, \\ Kista, Sweden \\ xavier.gelabert@huawei.com
}

\author{
R. Jäntti $i^{3}$ \\ ${ }^{3}$ Department of Communications and \\ Networking, School of Electrical \\ Engineering, Aalto University, Espoo, \\ Finland \\ riku.jantti@aalto.fi
}

\begin{abstract}
Future cellular networks need to support data hungry applications with enhanced data rates possibly via cell densification (ultra-small cells). The key objective of this work is to study the performance of a cellular network by taking into account the user mobility when LTE ultra-small cell network is deployed. Handover (HO) issues are analyzed considering various cell sizes, user speeds, and $\mathrm{HO}$ optimization related parameters. This study is very helpful to realize the problem of $\mathrm{HO}$ in $5 \mathrm{G}$ standalone network deployment as it shows the impact of LTE ultra-small cell deployment on the HO performance. A system level simulator is used to perform simulation and the results show that, as expected, the ultra-densification results in an increased number of HOs. Also, increasing the offset values have a significant impact on the HO reliability while changing the Time to Trigger (TTT) values has a less-significant impact.
\end{abstract}

Keywords-LTE, handover, ultra-small cells, 5G, performance evaluation, simulation.

\section{INTRODUCTION}

It is forecasted that the mobile data traffic will grow dramatically until the year 2021 especially due to the global adoption of smartphones and internet of things (IoT) devices. To deliver enhanced capacity and coverage, mobile operators will have to consider an alternative cellular network architecture to support billions of predicted connected devices [1]. The deployment of ultra-small cells is a key approach for future cellular networks to satisfy the growing data rate demands. However, out of many challenges of ultra-small cell deployment, $\mathrm{HO}$ has special importance.

What is a HO? When a user is active on a data session or a phone call while moving from one base station (BS) to another BS, such type of mobility is called connected mode mobility or a HO. The network has to provide its services to users on the move, at high speed within the vehicles or trains. In addition to providing high data rates, it is equally important to provide a reliable $\mathrm{HO}$ mechanism as this directly impacts the perceived quality of experience (QoE) of the user. Otherwise, the capacity gain achieved through densification will be counter-balanced with an increased number of HOs since the signaling overheads produced during the $\mathrm{HO}$ procedure reduces the user throughput [2]. The frequent HOs with ping-pong (PP) effect and $\mathrm{HO}$ failures also result in higher power consumption both at the network and user equipment (UE) side that further leads to high $\mathrm{CO}_{2}$ emissions [3]. In addition, if a high speed user goes through multiple HOs, the cumulative HO latency will result in a severe deterioration of the user's QoE [4].

With the above in mind, in this work, we study the performance of a cellular network by taking into account the user mobility when LTE ultra-small cell network is deployed. Various cell sizes, user speeds, and $\mathrm{HO}$ optimization related parameters are analyzed to see the impact of these parameters on $\mathrm{HO}$ rates, $\mathrm{HO}$ failure rate, $\mathrm{PP}$ rate and mean time between $\mathrm{HOs}$ (MTBH). We are focused to find optimum parameters out of the simulated cases that make the ultra-small cell deployment feasible. We believe this study is beneficial to realize the problem of $\mathrm{HO}$ in $5 \mathrm{G}$ standalone network deployment.

In related works, we can see that the study of the impact of varying the cell size on the $\mathrm{HO}$ procedure is not new, the impact of varying the inter site distance (ISD) (from $125 \mathrm{~m}$ to $1250 \mathrm{~m}$ ), user speed and $\mathrm{HO}$ related parameters on the $\mathrm{HO}$ procedure in an LTE network is addressed in [5]. It was found that increasing/decreasing the cell size after a certain limit brings the performance degradation in term of $\mathrm{HO}$ mainly due to the limitations in the uplink (UL). For low ISDs, the interference from closely located UEs, and for high ISDs, high path loss, results in severe degradation in performance. Similarly, the results in [2] show that a good compromise between the HO frequency and $\mathrm{HO}$ reliability can be achieved with the adjustment of $\mathrm{HO}$ related parameters namely, time to trigger (TTT) and A3 offset. Also, in heterogeneous networks (HetNets) deployment, the macro base station (BS) can be switched off to enable the power saving mode when the user speed is low and pico cell density is high to have comparable HO reliability [6].

The rest of the paper is organized as follows: Section II provides a brief overview of the HO mechanism in LTE. Section III discusses simulator modeling aspects. In Section IV, numerical results and discussion are presented. And finally, Section V provides conclusion.

\section{HANDOVER MECHANISM IN LTE}

An overall view of the HO procedure in LTE network is shown in Fig. 1 [7]. The HO procedure can be divided into seven steps. In the first step, the UE performs the downlink (DL) reference signal (RS) strength measurements from both the serving and the target eNBs. These measurements are processed at the UE side in step two. As the UE browses through the network, a target cell may become stronger than the source cell as shown in Fig. 2. If the target cell becomes stronger than the 
source cell by an offset value, called A3 offset, and this condition is maintained within a time defined by TTT, the measurement report is transmitted to the serving eNB in the third step. Based on the measurement report, the serving eNB sends a $\mathrm{HO}$ request message to the target eNB in the fourth step. When target eNB acknowledges the $\mathrm{HO}$ request, the serving eNB sends a HO command to the UE in the fifth step. In the sixth step, the UE starts accessing the target eNB via the random access channel (RACH) transmission. Upon successful synchronization with the target $\mathrm{eNB}$, the UE sends a $\mathrm{HO}$ confirm message to the target cell in the final step. In order to achieve a good compromise between $\mathrm{HO}$ reliability and $\mathrm{HO}$ frequency, HO optimization deals with the adjustment of the TTT and A3 offset [6].

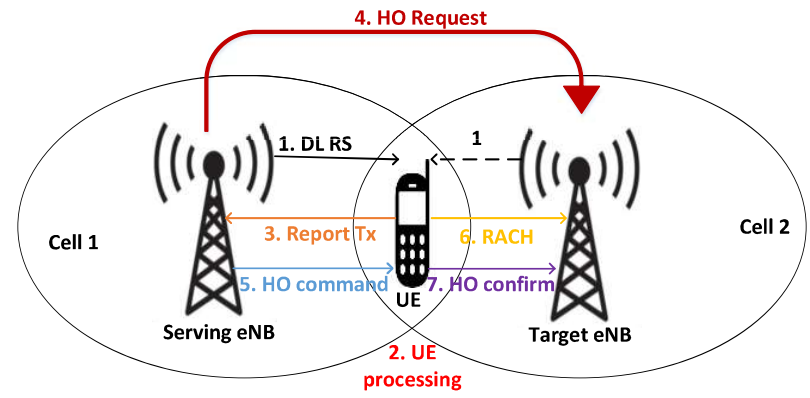

Fig. 1. LTE HO Procedure (adapted from [7]).

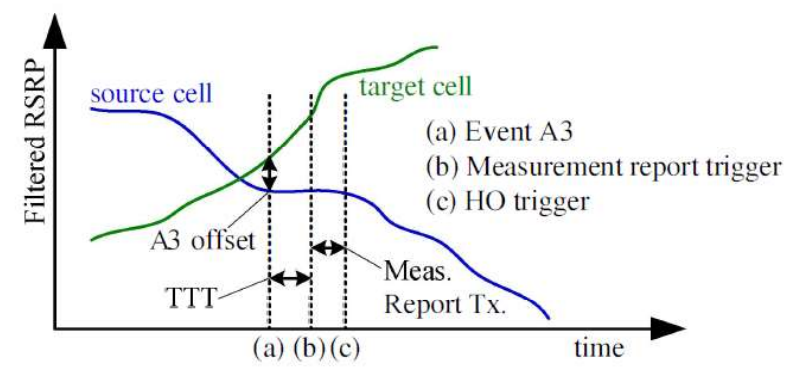

Fig. 2. UE measurement processing (adapted from [6]).

\section{SYSTEM MODEL}

A MATLAB based LTE system level simulator is used for the LTE ultra-small cell deployment with 16 tri-sectored eNBs, and 100 UEs that are placed across the scenario randomly with varying speed as shown in Fig. 3. Cell wrap-around feature is included in order to ensure fair interference conditions across the scenario. The inter-site distance (ISD), UE speed, TTT and A3 offset are varied from $\{20 \mathrm{~m}$ to $100 \mathrm{~m}\},\{3 \mathrm{~km} / \mathrm{h}$ to $120 \mathrm{~km} / \mathrm{h}\}$, $\{8 \mathrm{~ms}$ to $32 \mathrm{~ms}\}$ and $\{0.5 \mathrm{~dB}$ to $1.5 \mathrm{~dB}\}$, respectively. The results are analyzed considering the $\mathrm{HO}$ rate, $\mathrm{HO}$ failure rate, $\mathrm{PP}$ rate and mean time between HOs (MTBH). Although reducing the cell sizes increase the HO frequency as noted in [5], we will try to find best parameters match out of the simulated cases that will have less performance degradation in term of $\mathrm{HO}$. Moreover, radio link failures (RLFs) are also considered in the simulator. The main simulation assumptions are summarized in Table I.

\section{NumERICAL RESUlts AND Discussion}

In this section, we provide a numerical evaluation of the $\mathrm{HO}$ procedure with different conditions of cell sizes, UE speed, A3 offset and TTT. Fig. 4 shows the $\mathrm{HO}$ rate (measured in number of HOs in a second) against various simulated cell sizes and UE speeds. One trend of the graph shows that the increasing the user speed, increase the $\mathrm{HO}$ rate while the other trend shows that the ultra-small cell deployments (ISD $=20 \mathrm{~m}$ and $\mathrm{ISD}=40 \mathrm{~m}$ ) suffer from the higher number of $\mathrm{HO}$ rates. As the ISD increases (till ISD $=100 \mathrm{~m}$ ), the $\mathrm{HO}$ rate decreases to its minimal values out of the simulated cases.

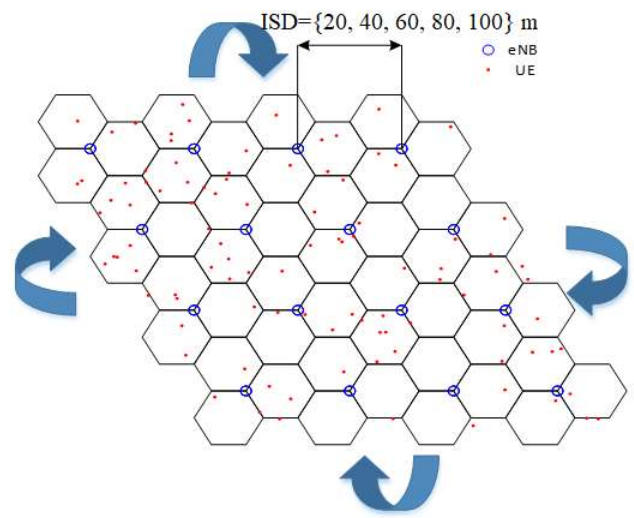

Fig. 3. Cell deployment scenario.

Table I Simulation parameters and assumptions.

\begin{tabular}{|c|c|}
\hline Feature & Imple mentation \\
\hline Network topology & $\begin{array}{c}\text { A hexagonal grid of } 16 \times 3=48 \text { cells (wrap-around } \\
\text { included) }\end{array}$ \\
\hline Inter-site distance & From the set $\{20,40,60,80,100\} \mathrm{m}$ \\
\hline System Bandwidth & $\begin{array}{c}B_{\text {sys }}=5 \mathrm{MHz} \text { (paired FDD), at carrier frequency } \\
\qquad f_{c}=2.1 \mathrm{GHz}\end{array}$ \\
\hline eNB DL power & $P_{e N B}=27 \mathrm{dBm}$ \\
\hline UE Power & $P_{U E}=23 \mathrm{dBm}$ \\
\hline Antenna patterns & 3D model specified in [8], Table A.2.1.1.2-2 \\
\hline Channel model & 6 tap model, Typical Urban (TU) \\
\hline Shadowing & $\begin{array}{c}\text { Log-normal Shadowing Mean } 0 \mathrm{~dB} \text {, Standard } \\
\text { deviation: } 10 \mathrm{~dB}\end{array}$ \\
\hline Propagation model & $L=140.7+36.7 \log _{10}(R), R$ in $\mathrm{km}[8]$ \\
\hline UE speed & from the set $\{3,30,60,120\} \mathrm{km} / \mathrm{h}$ \\
\hline $\begin{array}{l}\text { RLF detection by L1 } \\
\text { of UE }\end{array}$ & $\begin{array}{l}\text { T310 }=1 \mathrm{~s}, \mathrm{~N} 310=1, \mathrm{~N} 311=1 \text { as specified in [9] } \\
\mathrm{Q}_{\mathrm{in}}=-4.8 \mathrm{~dB} ; \mathrm{Q}_{\text {out }}=-7.2 \mathrm{~dB} \text { as specified in [10] }\end{array}$ \\
\hline HO parameters & $\mathrm{TTT}=\{8,16,32\} \mathrm{ms}, \mathrm{A} 3$ offset $=\{0.5,1,1.5\} \mathrm{dB}$. \\
\hline
\end{tabular}

Next, we draw our attention to $\mathrm{HO}$ failure ratio to find the percentage of HOs that could not be completed seamlessly. Fig. 5 shows the HO failure ratio against considered ISDs and UE speeds. The HO failure ratio for low ISD is higher because increasing the ultra-small cell density in a specific area increases the number of potential interferers which degrades the SINR and hence causes more HO failures. Also, for low ISD, high speeds will cause the user moving away from the source cell thus have the highest HO failure ratio. More interestingly, both $3 \mathrm{~km} / \mathrm{h}$ and $30 \mathrm{~km} / \mathrm{h}$ speed values have almost the same number of $\mathrm{HO}$ failures. 


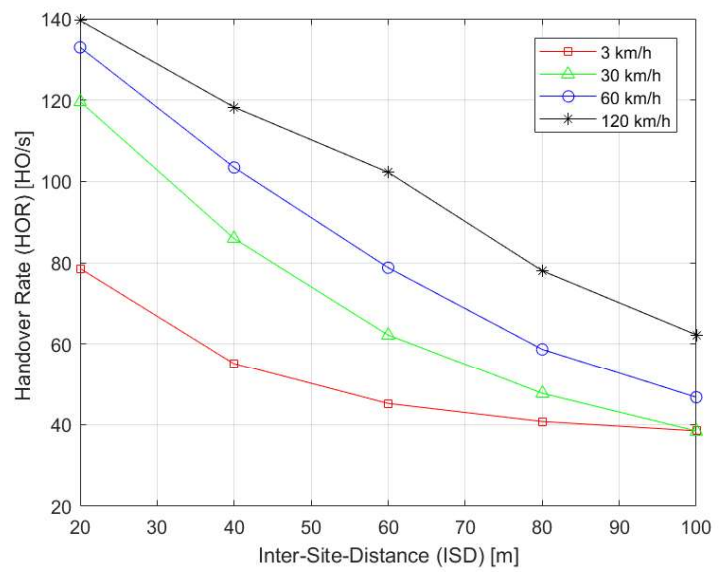

Fig. 4. HO rate against various cell sizes and speed values at fixed TTT=32 $\mathrm{ms}$ and offset $=1 \mathrm{~dB}$.

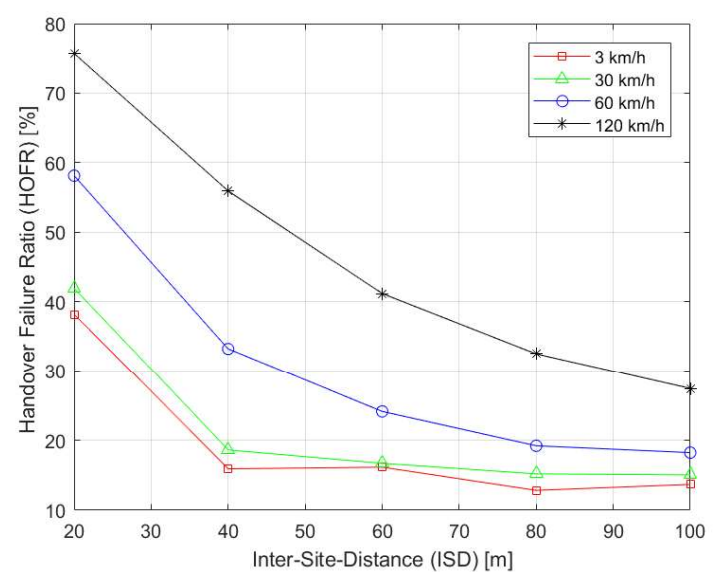

Fig. 5. HO failure ratio in $\%$ against various ISD and speed values at fixed TTT $=32 \mathrm{~ms}$ and offset $=1 \mathrm{~dB}$.

Fig. 6 shows the HO failure ratio breakdown per failure type (F0 to F7). It is clear from the graph that the HO failures impacts the most on UL transmissions, i.e. measurement report (F2 in red) and HO confirm transmission error (F7 in yellow). Another failure type, T310 expiry before the HO command is received (F4 in blue), is higher especially at high speeds. This is because the UEs that are able to transmit the measurement report move out of the serving cell and thus the HO command sent by the serving eNB cannot be received at the UE side. Noteworthy, LTE implements the cell-reselection mechanism upon a $\mathrm{HO}$ failure to recover the UE connection but this comes at the cost of increased interruption time and delays.

Fig. 7 shows the PP rate against simulated cases of ISDs and UE speeds. A PP event is defined as a HO from serving eNB to the target eNB followed by another $\mathrm{HO}$ to the original source eNB which happens within a predefined time duration that is 3 seconds in our simulation scenario. More interestingly, the lowest UE speed cases experience the highest PP rate because there are more chances for the UE to come back to the serving cell with in the predefined time interval. For high speed values $\{60,120\} \mathrm{km} / \mathrm{h}$, the PP rate is lower for low ISD then it increases till the medium ISD arrives and then again start decreasing for high ISDs. This is because low ISDs have a higher number of potential interferers and for high ISD, the UEs located at the cell edge will transmit at maximum power and thus create excessive interference to the neighboring cells. As a consequence, $\mathrm{HO}$ failures may occur and the UEs try to reconnect to the source eNB even if it is not the most suitable $\mathrm{eNB}$ at that time.

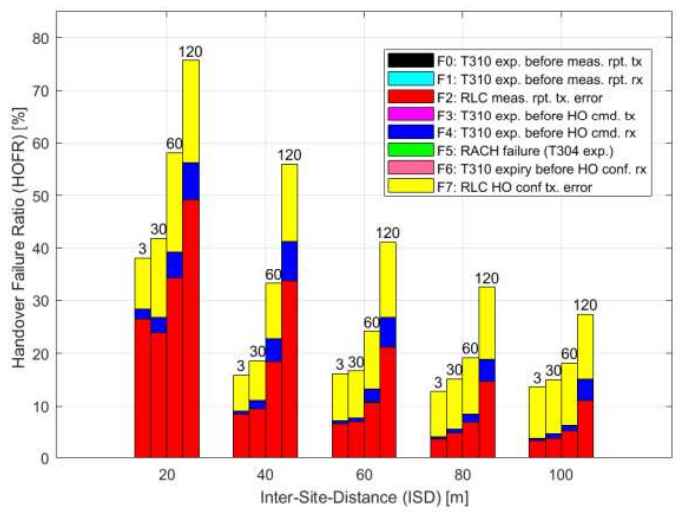

Fig. 6. HO failure ratio in $\%$ breakdown per HO failure type against different ISD and speed values at fixed TTT $=32 \mathrm{~ms}$ and offset $=1 \mathrm{~dB}$.

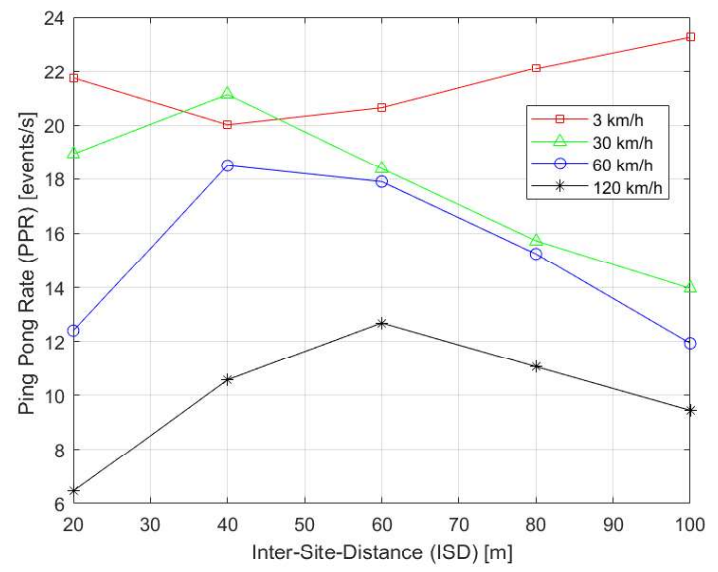

Fig. 7. PP rate against various ISDs and UE speeds at fixed TTT=32 $\mathrm{ms}$ and offset $=1 \mathrm{~dB}$.

A mean elapsed time between HOs against ISD and different UE speeds is shown in Fig. 8. One trend of the graph shows that increasing the ISD, increases the MTBH (less frequent HOs) while the next trend shows that the high speed values have the lower MTBH (more frequent HOs). 


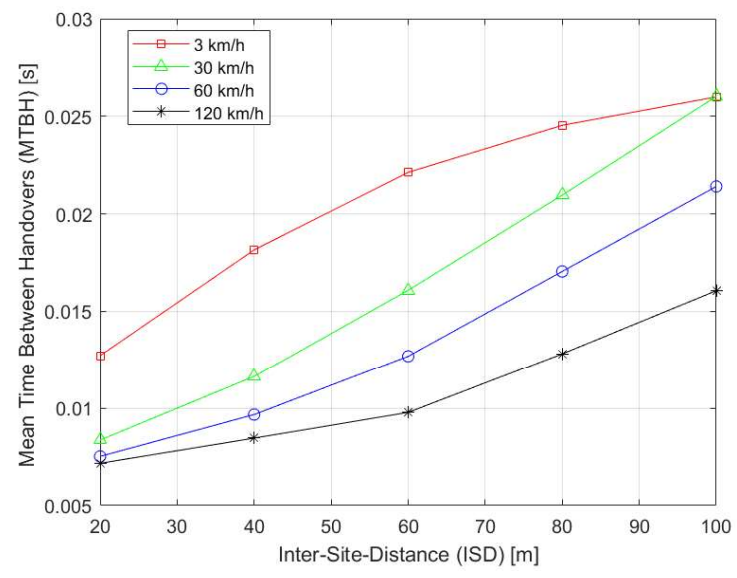

Fig. 8. MTBH against ISDs and different UE speed values at fixed TTT=32 $\mathrm{ms}$ and offset $=1 \mathrm{~dB}$.

HO interruption time (HOIT) is defined as the time duration whereby the user plane is unable to deliver the packet to and from the UE. It mainly includes the time during which the UE performs initial access to the target cell, i.e. cell synchronization and random access procedures. Fig. 9 shows the CDF of HOIT at different speed values. We observe that the lower speed benefits from the lower HOIT since fewer HO failures are noted for this case (see Fig. 5).

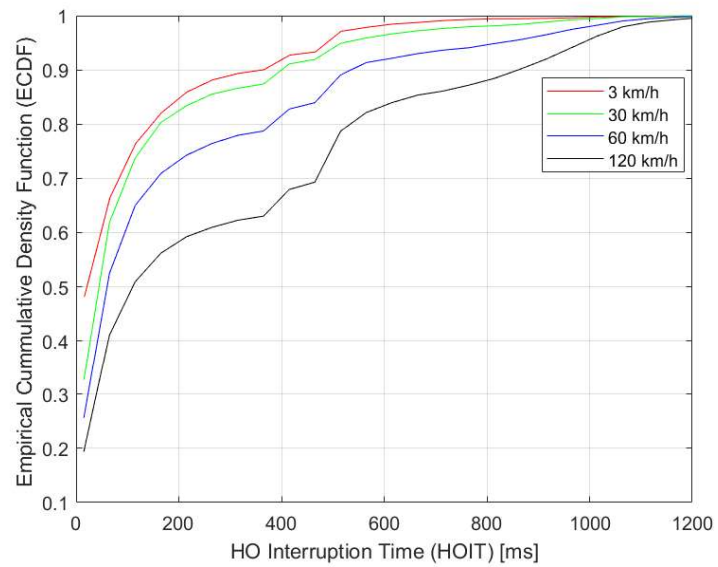

Fig. 9. Empirical Cumulative density function (CDF) of the HO Interruption Time against different $\mathrm{UE}$ speed values at $\mathrm{ISD}=40 \mathrm{~m}$, TTT $=32 \mathrm{~ms}$ and offset= $1 \mathrm{~dB}$.

The impact of varying the offset values on $\mathrm{HO}$ rate and the $\mathrm{HO}$ failure rate is shown in Fig. 10 and Fig. 11 respectively. It is clear from the graphs that increasing the offset values, reduces the $\mathrm{HO}$ rate as well as the $\mathrm{HO}$ failure rate. The results show that the highest value of the offset $(1.5 \mathrm{~dB}$ case $)$ is the optimum out of the simulated cases.

The impact of varying the TTT values on $\mathrm{HO}$ rate and the $\mathrm{HO}$ failure rate is shown in Fig. 12 and Fig. 13 respectively. It is clear from the graphs that varying the TTT values have a less significant impact on $\mathrm{HO}$ rate and $\mathrm{HO}$ failure rate. So, choosing the lowest value of TTT $(8 \mathrm{~ms})$ is optimum to trigger the $\mathrm{HO}$ early and reduce the HO latency.

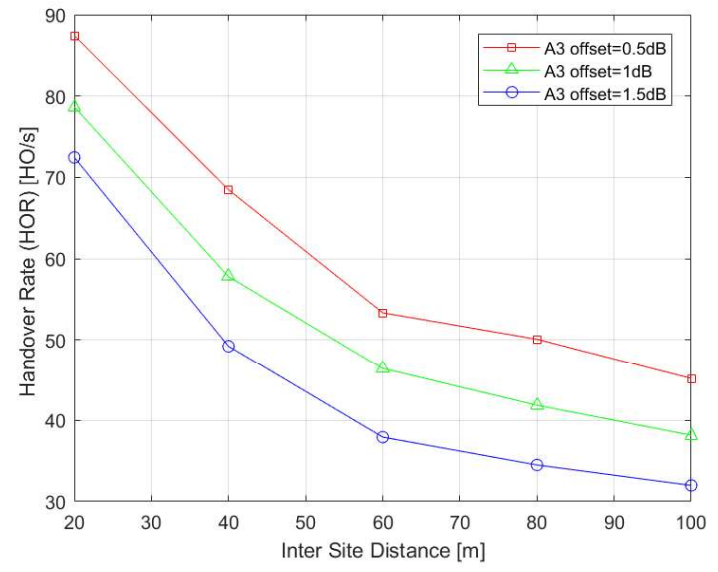

Fig. 10. Impact of varying offset values $\{0.5,1,1.5\} \mathrm{dB}$ on $\mathrm{HO}$ rate at contant speed $=3 \mathrm{~km} / \mathrm{h}$ and $\mathrm{TTT}=8 \mathrm{~ms}$.

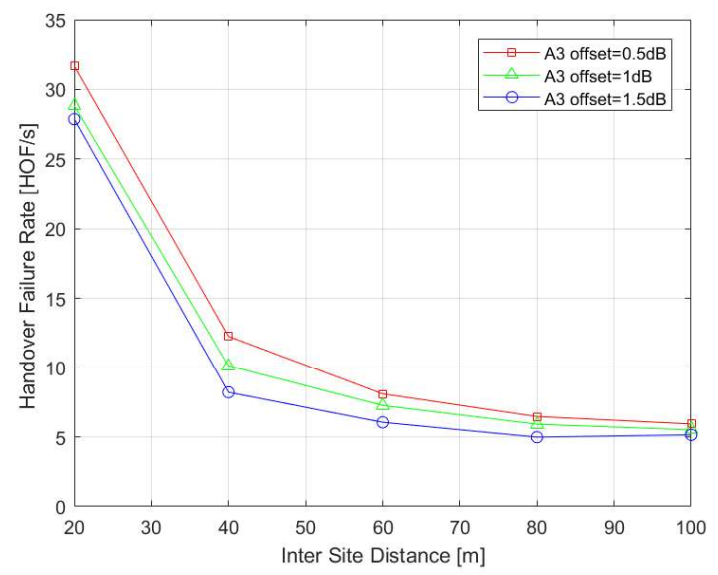

Fig. 11. Impact of varying offset values $\{0.5,1,1.5\} \mathrm{dB}$ on $\mathrm{HOF}$ rate at contant speed $=3 \mathrm{~km} / \mathrm{h}$ and $\mathrm{TTT}=8 \mathrm{~ms}$.

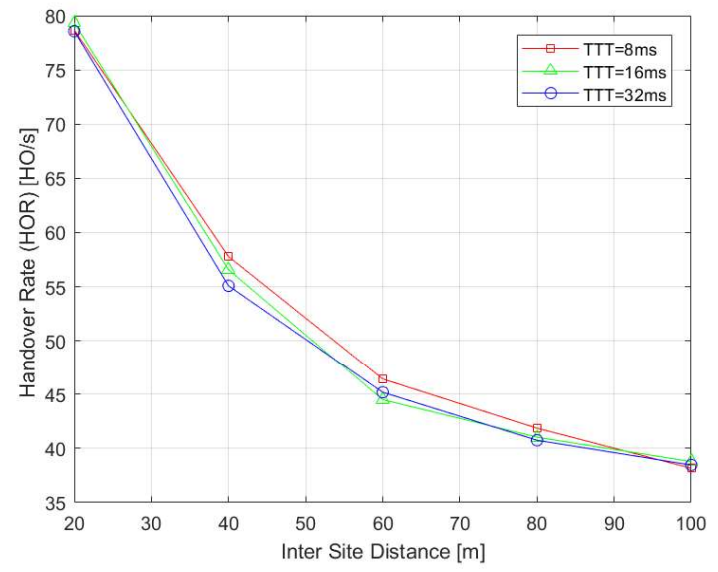

Fig. 12. Impact of varying TTT values $\{8,16,32\} \mathrm{ms}$ on $\mathrm{HO}$ rate at contant speed $=3 \mathrm{~km} / \mathrm{h}$ and offset $=1 \mathrm{~dB}$. 


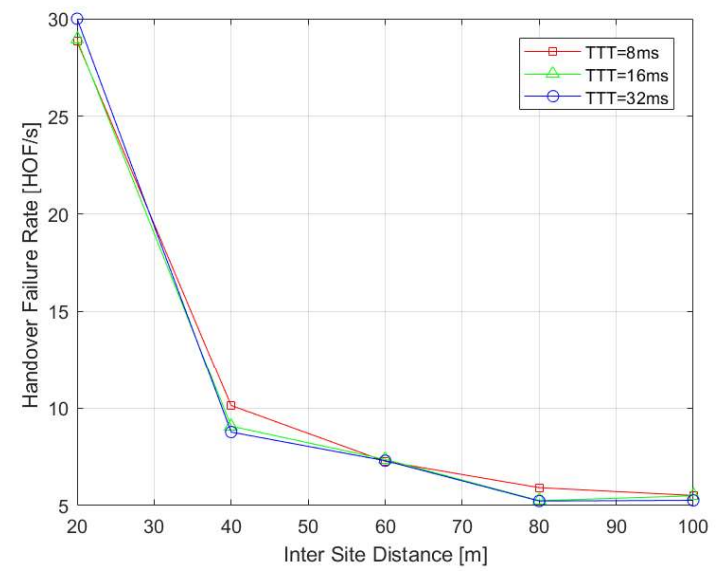

Fig. 13. Impact of varying TTT values $\{8,16,32\} \mathrm{ms}$ on $\mathrm{HO}$ failure rate at contant speed $=3 \mathrm{~km} / \mathrm{h}$ and offset $=1 \mathrm{~dB}$.

\section{CONCLUSIONS}

A simulation analysis is presented to analyze the $\mathrm{HO}$ problem in LTE ultra-small cell deployments. This work highlights some $\mathrm{HO}$ issues and identifies research items that need further investigation for the implementation of $5 \mathrm{G} N R$ standalone architecture in the future. We observe that reducing the cell sizes causes performance degradation in terms of $\mathrm{HO}$ mainly due to UL limitations, also noted in [5]. We show that the HO failures are mostly concentrated due to UL transmission error, mainly measurement report and $\mathrm{HO}$ confirm transmission errors. We find the PP rate analysis more interesting as the lowest speed has the highest PP rate while the high speed has the lower PP rate (with high HO failures) that brings a new finding for ultra-small cell deployment compared to our work in [5]. Overall, the PP rate for ultra-small cell deployment is higher when compared to larger cells [5]. We can say that suitable ISD value is $80 \mathrm{~m}$ out of the simulated cases, since a compromised $\mathrm{HO}$ rate, $\mathrm{HO}$ failures and $\mathrm{PP}$ rate are noted for this case. We also observe that varying the offset values have a significant impact on both the HO rates and HO failure rate but changing the TTT values have a less significant impact. We argue that an offset value of $1.5 \mathrm{~dB}$ and TTT value of $8 \mathrm{~ms}$ (out of the simulated cases) is an optimum case to trigger the $\mathrm{HO}$ early and thus reduce the HO rate and HOF rate in LTE ultra-small cell deployments. We suggest using a low TTT value for 5G ultra-small cell deployments to improve the HO performance. In the future, we will consider the mobile small cell operation (a use case of 5G) in the simulation scenario.

\section{ACKNOWLEDGMENT}

This project has received funding from the European Union's H2020 research and innovation program under grant agreement H2020-MCSA-ITN- 2016-SECRET 722424 [11].

\section{REFERENCES}

[1] Huawei and Ericsson, "Small Cell Network White Paper", GSA white paper, Nov. 2016.

[2] R. Arshad, H. Elsawy, S. Sorour, T. Y. Al-Naffouri and M. S. Alouini, "Handover Management in 5G and Beyond: A Topology Aware Skipping Approach", IEEE Access, vol. 4, pp. 9073-9081, Dec 2016.

[3] M. Tayyab, G. P. Koudouridis, X. Gelabert and R. Jäntti, "Signaling Overhead and Power Consumption during Handover in LTE”, IEEE WCNC conference 2019. [Accepted]

[4] T. Bilen, B. Canberk, and K. R. Chowdhury, "Handover Management in Software-Defined Ultra-Dense 5G Networks", IEEE Network, vol. 31, pp. 49-55, Aug 2017.

[5] M. Tayyab, G.P. Koudouridis, X. Gelabert "A Simulation Study on LTE Handover and the Impact of Cell Size". Broadband Communications, Networks, and Systems. (BROADNETS 2018).

[6] X. Gelabert, G. Zhou and P. Legg, "Mobility Performance and Suitability of Macro Cell Power-Off in LTE Dense Small Cell HetNets", IEEE 18th International Workshop on Computer Aided Modeling and Design of Communication Links and Networks (CAMAD), pp. 99-103, 2013.

[7] 3GPP TS 36.300, "(E-UTRA) and (E-UTRAN); Overall description; Stage 2 (Release 15)" V15.0.0, Section 10, pp. 93-143, Dec. 2017.

[8] 3GPP TR 36.814, "Further advancements for E-UTRA physical layer aspects (Release 9),"V9.0.0, Mar 2011.

[9] 3GPP TS 36.331, "E-UTRA Radio Resource Control (RRC); Protocol specification (Release 9)", v9.2.0, Mar 2010.

[10] 3GPP TS 36.133, "Requirements for support of radio resource management (Release 9)," v9.15.0, Mar 2013.

[11] J. Rodriguez et al., "SECRET - Secure Network Coding for Reduced Energy Next Generation Mobile Small cells", IEEE Internet Technologies and Applications (ITA) conference, pp. 329-333, 2017. 